\title{
Orchids of the State Park of Serra do Tabuleiro, Southern Brazil
}

\author{
Maurício Lenzi ${ }^{1 *}$, Josy Zarur de Matos ${ }^{2}$, Angelo Martins Fraga ${ }^{3} \&$ Manuel B. Crespo ${ }^{4}$ \\ ${ }^{1}$ Capes Foundation, Ministry of Education of Brazil, Cx. postal 250, Brasília, DF, CEP: 70040-020, Brazil; mlenzi34@hotmail.com \\ ${ }^{2}$ Museu de Ciências Naturais, Fundação Zoobotânica do Rio Grande do Sul, Rua: \\ Dr. Salvador França, 1427, Porto Alegre, RS, Cep: 90.690-000, Brazil; josyzarur@gmail.com \\ ${ }^{3}$ Instituto Federal de Educação, Ciência e Tecnologia de Santa Catarina, Campus Florianópolis, \\ Av. Mauro Ramos, 950, Centro - Florianópolis, SC, CEP: 88020-300, Brazil; angelofraga@yahoo.com.br \\ ${ }^{4}$ dCARN \& CIBIO (Instituto de la Biodiversidad), Universidad de Alicante, P.O. Box 99, E-03080 Alicante, Spain; crespo@ua.es
}

\begin{abstract}
Lenzi, M., de Matos, J.Z., Fraga, A.M. \& Crespo, M.B. 2015. Orchids of the State Park of Serra do Tabuleiro, Southern Brazil. Anales Jard. Bot. Madrid 72(2): e020.

Orchidaceae show a high diversity of species in Brazil, especially in the Atlantic Forest Biome. Over the course of a 12-month study in the State Park of Serra do Tabuleiro in Southern Brazil, collections were made of orchids occurring in areas of restinga and riparian vegetation. A total of 92 orchid species were registered, belonging to three subfamilies and 51 genera. Octomeria was the best represented genus with ten species. Among all collections, two species are new records for Santa Catarina state, and 20 species is reported for the first time in the studied region. Regarding to conservation, 17 species (19\%) are currently included with different threat labellings in any of the red lists of threatened plant species of Brazil. Among the total of orchids registered, 55 species (59\%) are characteristic holoepiphytes, 47 species $(51 \%)$ are restricted to the Atlantic Forest, 77 species (84\%) occur in the riparian vegetation, and only nine species $(10 \%)$ are restricted to restinga vegetation. The remarkable richness of orchids found in the present study highlights the importance for conservation the studied forest remnants. The historical of environmental degradation of the restinga vegetation can be negatively influencing the current floristic composition of the orchid community.
\end{abstract}

Keywords: Orchidaceae, Atlantic Forest, biogeographic boundaries, forest conservation, fragmentation, restinga, riparian forest.

\section{INTRODUCTION}

Orchidaceae are usually considered to be one of the widest families of Angiosperms, with around 20000 species (Dressler, 1993). According to Barros \& al. (2015), the family is well represented in Brazil with 238 genera and about 2553 species, 1636 of which are endemic to the country.

In the state of Santa Catarina, Southern Brazil, the family is still poorly known. In the most recent checklist of Orchidaceae published for that state, Klein \& al. (1978) listed 295 species for the whole Florianópolis Island plus nine neighbouring municipalities.

The State Park of Serra do Tabuleiro covers an area of $87405 \mathrm{ha}$, which represents approximately $1 \%$ of the state of Santa Catarina, and is very close to Florianópolis Island. It reaches nine municipalities and several coastal islands. All these different areas comprise a great diversity of natural environments, ranging from coastal environments to inland habitats that represent five of the six phytogeographic regions found in the state. The park is still

\section{Resumen}

Lenzi, M., de Matos, J.Z., Fraga, A.M. \& Crespo, M.B. 2015. Orquideas del Parque Estatal de Serra do Tabuleiro, S de Brasil. Anales Jard. Bot. Madrid 72(2): e020.

Las Orchidaceae presentan una gran diversidad de especies en Brasil, especialmente en el Bioma de la Mata Atlântica. Tras estudiar durante un año el Parque Estatal de Serra do Tabuleiro, se realizaron numerosas recolecciones de las orquídeas presentes en áreas de restinga y bosque de ribera. Se registraron 92 especies de orquídeas, pertenecientes a 3 subfamilias y 51 géneros. Octomeria fue el género mejor representado, con 10 especies. De los táxones observados, 2 especies son novedad para el estado de Santa Catarina, y 20 especies se citan por primera vez en la región estudiada. Respecto a su conservación, 17 especies (19\%) están actualmente incluidas con distintos grados de amenaza en alguna de las listas rojas de flora amenazada de Brasil. De todas las orquídeas observadas, 55 especies (59\%) son holoepífitos típicos, 47 especies (51\%) se restringen a la Mata Atlântica, 77 especies (84\%) crecen en los bosques ribereños, y sólo 9 especies (10\%) se restringen a la vegetación de restinga. La destacable riqueza de orquídeas evidenciada en el presente estudio viene a destacar la importancia de conservar los fragmentos de bosque estudiados. El registro histórico de la degradación ambiental de la vegetación de restinga puede influir negativamente a la composición florística de la comunidad de orquídeas de estas zonas.

Palabras clave: Orchidaceae, Mata Atlântica, límites biogeográficos, conservación florestal, fragmentación, restinga, bosque de ribera.

considered the southern boundary for many species with tropical distribution, thus constituting an important phytogeographic barrier (Klein, 1978; 1980; 1981). It comprises areas of Atlantic Forest (Mata Atlântica) formed by riparian and restinga vegetation.

The Atlantic Forest is considered as one of the richest biomes of the planet in biodiversity, and also one of the most severely threatened ecosystems; less than $10 \%$ of its original area remains well conserved (Galindo-Leal \& Câmara, 2005). The state of Santa Catarina is totally included into this biome and at present possesses only $23.5 \%$ of its forest remnants in good estate of conservation (Fundação SOS Mata Atlântica, 2013). Studies with vascular species undertaken on plant communities growing close to water courses (riparian vegetation), although still scarce, show that they constitute very diverse ecosystems due to their high environmental heterogeneity (Kageyama \& Gandara, 2000). According to Battilani \& al. (2005), riparian vegetation is crucial to maintain the local ecosystem integrity and it plays an important role for conservation of high diversity sites. 
Littoral plant communities, that constitute the restinga vegetation, occur on the coastal flats and possess a wide diversity of habitats (Klein, 1980; Scherer \& al., 2005). Recent studies (Fraga \& Peixoto, 2004, Rocha \& Waechter, 2010) highlighted the species richness of orchids in this ecosystem. However, Falkenberg (1999) and Scherer \& al. (2005) noted that the impact of human activities has caused strong degradation and fragmentation of the coastal environments, hampering the current understanding of patterns of both abundance and distribution of the local flora.

These factors together increase the threat on the orchid flora of the State Park of Serra do Tabuleiro and also in the state of Santa Catarina as a whole, and point out the importance to take urgent steps for the study and maintenance of biodiversity in these areas. An inventory of the orchids of the State Park of Serra do Tabuleiro may help to determine species subjected to some threat and to prepare future red lists of species of threatened flora for Santa Catarina and other regions of Brazil.

The objectives of the present study were: 1) to record the diversity of Orchidaceae in remnants of restinga and riparian vegetation in the Atlantic Forest, and 2) to update and depict the current geographic distribution of these species, threat category in red lists and their life forms, as the basic step for future studies on conservation of local biodiversity.

\section{MATERIAL AND METHODS}

\section{Study region}

The State Park of Serra do Tabuleiro (Fig. 1) is located in the central part of the coast of Santa Catarina state (Lat. $27^{\circ} 41^{\prime} 09^{\prime \prime} \mathrm{S}$ to $28^{\circ} 12^{\prime} 42^{\prime \prime} \mathrm{S}$, Long. $48^{\circ} 49^{\prime} 20^{\prime \prime} \mathrm{W}$ to $\left.48^{\circ} 25^{\prime} 08^{\prime \prime} \mathrm{W}\right)$, to $\mathrm{SW}$ of Florianópolis Island. The main orientation is on N-S direction and lesser in E-W direction, following the mountain ranges of Cambirela, Tabuleiro and Capivari. The altitude gradient ranges from the sea level in the coastal areas, up to over $1200 \mathrm{~m}$ in Serra do Tabuleiro (Klein, 1978; 1981).

The climate is characterized by mild and rainy summers, and humid and warm winters. The average annual rainfall is $1600 \mathrm{~mm}$ and the annual average temperature is $20.3^{\circ} \mathrm{C}$ (INMET, 2011).

The present study covered a total area of $34400 \mathrm{~m}^{2}$ distributed along eight transects in eight sites (Appendix 1): two of them in restinga vegetation $\left(20000 \mathrm{~m}^{2}\right)$, and six of them in riparian vegetation $\left(14400 \mathrm{~m}^{2}\right)$. These transects were characterized according to their location, morphology and vegetation type, as indicated below:

i) Site 1, and Site 2, (Fig. 1 \& Appendix 1), both transects were located so as to avoid sites disturbed by fires that occurred in the region between 2001 and 2008, as surveyed by Pereira \& al. (2009). Sampling was settled with $100 \mathrm{~m}$ side squares, distancing a maximum of $100 \mathrm{~m}$ of the beach line and totalling $20000 \mathrm{~m}^{2}$ of area. The areas are covered by restinga vegetation, with herbaceous and woody layers. This vegetation occurs exclusively in coastal flats, which are geomorphological units constituted by marine sediments, along the Brazilian coast at altitudes varying from sea level up to $30 \mathrm{~m}$ towards inland the continent (Klein, 1980). According to Falkenberg (1999), the herbaceous and subshrub layers of restinga vegetation are constituted mainly by small plants that endure higher luminosity and stronger sea influence, therefore comprising plant communities closer to the sea. The shruby layer includes taller species (between 1 to $5 \mathrm{~m}$ in height).

ii) The other six transects (Fig. 1 \& Appendix 1) were located along both sides of several rivers and around small river islands. Site 3 , and Site 8, each with an extension of $800 \mathrm{~m}$ and a total area of $3200 \mathrm{~m}^{2}$. Site 4, Site 5 , Site 6, and Site 7, each with an extension of $500 \mathrm{~m}$ and a total area of $4000 \mathrm{~m}^{2}$. Morphologically, all these areas are formed by deep river valleys with an intense dissection, with very steep slopes and with a drainage network of waterfalls. The Atlantic Forest is represented on the slopes of the Serra do Tabuleiro, between 30 and $400 \mathrm{~m}$ altitude; forming part of a set of mountain plant communities with physiognomic variations, which is known as Floresta Ombróflia Densa and which constituting the largest part of forest diversity in the region (Klein, 1980). The riparian vegetation is a characteristic type of plant community found along the river courses, which vary according to ecological and biogeographic features of territories, their altitudes, and the dominant plant community of each site (Rodrigues \& Gandolfi, 2001).

\section{Plant survey}

Field work was carried out between March and October 2010. Orchid specimens were collected from soil, rocks and phorophytes (up to $5 \mathrm{~m}$ in height) in restinga (herbaceous, subshrubby and shrubby layers), and in riparian vegetation. All samples were deposited in the herbaria $\mathrm{ABH}$ and FLOR (acronyms according to Thiers, 2015).

Species identification was achieved by using specialized literature, namely Pabst \& Dungs $(1975 ; 1977)$, Miller \& Warren (1996), Stancik (2004) and Miller \& al. (2006). Herbarium materials from FLOR, FURB, HBR, ICN and MBM (acronyms according Thiers, 2015), were also used for comparison. Authors of plant names as well as the nomenclatural update of names follow IPNI (2015). The systematic position of the genera of Orchidaceae is according to Chase $\&$ al. (2003). However, Dendrobiinae and Bulbophyllinae are circumscribed following Dressler (1993).

Geographic distributions of species were updated through the list of Klein \& al. (1978) and Barros \& al. (2015).

Species classification into the different categories follows the existing information from the official lists of threatened plant species for the states (Santa Catarina: Klein, 1990; Paraná: SEMA, 1995; Rio Grande do Sul: SEMA, 2014; São Paulo: SEMA, 2004; Espírito Santo: IPEMA, 2007) and the whole country ( Martinelli \& Moraes, 2014). When categories of two or more lists were in conflict, the most recent (since updated) labelling was selected. Only those taxa identified to species rank were used to outline geographic distribution and threat categories.

The Ecological Category (EC) of each species was determined by direct visual observations in the field as being: (a) characteristic holoepiphyte (HLC), which occurs on phorophytes; (b) facultative holoepiphyte (HLF), based on the 

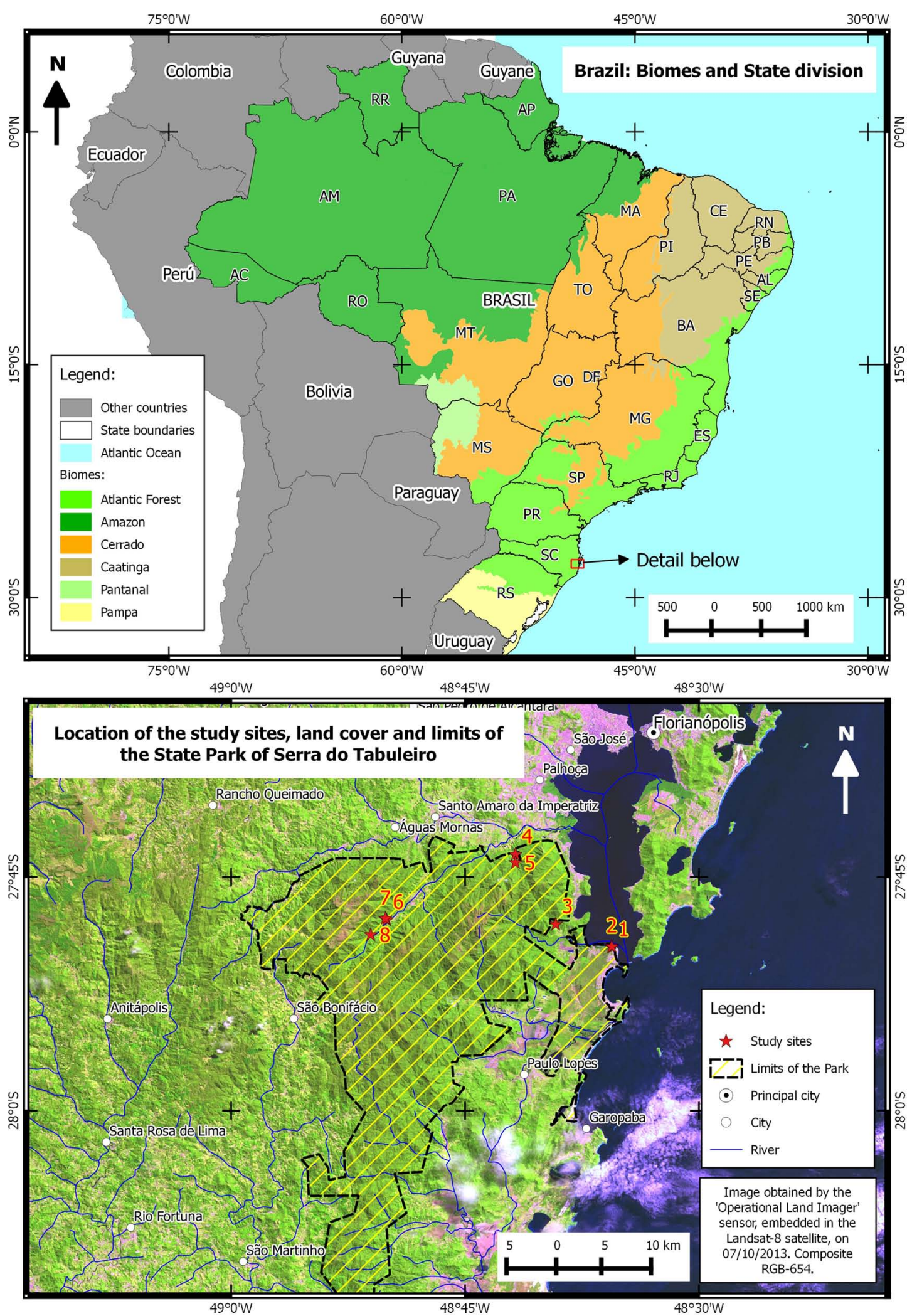

Fig. 1. Location of the study sites, land cover and limits of the State Park of Serra do Tabuleiro, Santa Catarina, Brazil. Source: compiled from IBGE (2014), MMA (2015) and USGS (2015).

type of relationship with the type of substrate (phorophytes or rocks); (c) hemiepiphyte (HEM) (Benzing 1990); and (d) terrestrial (TER) (Dressler, 1981).
All collections in the State Park of Serra do Tabuleiro were authorized by the Fundação do Meio Ambiente de Santa Catarina - FATMA (Aut. Nº02/2010/GERUC/DPEC). 
Thematic cartography was made with QGIS (for geographic information system and treatment of orbital images). The location of transects on the field was determined using a GPS.

\section{RESULTS}

\section{Species richness}

A total of 92 taxa of Orchidaceae were identified, which belong to 51 genera distributed in three subfamilies, 11 tribes and 13 subtribes (Table 1). Subfamily Epidendroideae was the most representative with 41 genera $(80 \%)$, followed by Orchidioideae with seven genera (16\%), Vanilloideae with two genera (4\%) (Table 1). Of the 92 recorded taxa, three were not identified at the species rank: Campylocentrum sp., Pelexia $\mathrm{sp}$. and Vanilla sp. (probably an exemplar of $V$. dietschiana Edwall), as well as an adult plant of small size $( \pm 110 \mathrm{~cm})$ that showed flower remnants insufficient for identification.

Octomeria was the best represented genus with 10 species, followed by Epidendrum with six especies; Bifrenaria and Stelis each with five species; Anathallis and Maxillaria each with four species; Gomesa, Pabstiella and Polystachya each with three species; Acianthera, Brasiliorchis, Campylocentrum, Christensonella, Dichaea, Dryadella, Phymathidium and Vanilla each with two species. The remaining 33 genera were represented by one species (Table 2).

\section{Geographic distribution and conservation of endangered species}

With regard to the geographic distribution in the states of Brazil, all the specimens recorded to species rank $(n=89)$ occur in two or more states of the country (Table 2).

Some species $(n=6 ; 7 \%)$ can be found in at least four phytogeographic domains, as is the case of E. secundum, L. nervosa, O. grandiflora, O. maculata, P. concreta and S. lanceolata. However, the Atlantic Forest (MA) has the largest number of exclusive species ( $\mathrm{n}=47 ; 53 \%)$. Out of 89 taxa identified to species rank, 33 species $(37 \%)$ are distributed through the state of Santa Catarina, but they do not reach the southern state of Rio Grande do Sul (Table 2).

Based on available checklists (Klein \& al., 1978; Barros \& al., 2015) and data from herbaria, of a total of 89 taxa identified to species rank, two species are recorded for the first time in the state of Santa Catarina, and 20 species are new for the municipalities of Palhoça and Santo Amaro da Imperatriz (Table 2), which are partially included within the limits of the State Park of Serra do Tabuleiro (Fig. 1).

According to all official lists of the Brazilian states, 17 species $(19 \%)$ are labelled in some degree of threat: six species $(7 \%)$ as endangered (ED), seven species $(8 \%)$ as vulnerable (VU), two species $(2 \%)$ as critically endangered (CE) and two species $(2 \%)$ as presumably extinct (Table 2 ).

\section{Habitat selectivity and ecological category}

As for the distribution of species $(\mathrm{n}=92)$ in the different vegetation types, the largest number of species $(n=77 ; 84 \%)$ occurred exclusively in riparian vegetation (RP), while only nine species $(10 \%)$ exclusively in the restinga vegetation (RE) (Table 2 \& Fig. 2). However, some species ( $\mathrm{n}=6 ; 7 \%$ ), namely
Table 1. Systematic position of the genera of Orchidaceae recorded in the State Park of Serra do Tabuleiro, Santa Catarina, Brazil, according to Chase \& al. (2003). Dendrobiinae and Bulbophyllinae accord with circumscription by Dressler (1993)

\begin{tabular}{|c|c|c|c|}
\hline Subfamilies & Tribe & Subtribe & Genus \\
\hline \multirow[t]{41}{*}{ Epidendroideae } & Cymbidieae & Catasetinae & Cyrtopodium \\
\hline & & Eulophiinae & Oeceoclades \\
\hline & & Maxillariinae & Bifrenaria \\
\hline & & & Brasiliorchis \\
\hline & & & Christensonella \\
\hline & & & Heterotaxis \\
\hline & & & Maxillaria \\
\hline & & & Ornithidium \\
\hline & & & Rhetinantha \\
\hline & & Oncidiinae & Gomesa \\
\hline & & & Ornithocephalus \\
\hline & & & Phymatidium \\
\hline & & & Rodriguezia \\
\hline & Dendrobieae & Dendrobiinae & Bulbophyllum \\
\hline & Epidendreae & Laeliinae & Brassavola \\
\hline & & & Cattleya \\
\hline & & & Encyclia \\
\hline & & & Epidendrum \\
\hline & & Ponerinae & Isochilus \\
\hline & & Pleurothallidinae & Acianthera \\
\hline & & & Anathallis \\
\hline & & & Barbosella \\
\hline & & & Dryadella \\
\hline & & & Lepanthopsis \\
\hline & & & Myoxanthus \\
\hline & & & Octomeria \\
\hline & & & Pabstiella \\
\hline & & & Phloeophila \\
\hline & & & Platystele \\
\hline & & & Pleurothallis \\
\hline & & & Specklinia \\
\hline & & & Stelis \\
\hline & & & Trichosalpinx \\
\hline & Sobralieae & & Elleanthus \\
\hline & & Zygopetalinae & Dichaea \\
\hline & & & Paradisanthus \\
\hline & Malaxideae & & Liparis \\
\hline & & & Malaxis \\
\hline & Triphoreae & & Psilochilus \\
\hline & Vandeae & Angraecinae & Campylocentrum \\
\hline & & Polystachyinae & Polystachya \\
\hline \multirow[t]{7}{*}{ Orchidoideae } & Orchideae & Orchidinae & Habenaria \\
\hline & & Spiranthinae & Eurystyles \\
\hline & & & Lankesterella \\
\hline & & & Mesadenella \\
\hline & & & Pelexia \\
\hline & & & Sacoila \\
\hline & & & Serapias \\
\hline \multirow[t]{2}{*}{ Vanilloideae } & Pogonieae & & Cleistes \\
\hline & Vanilleae & & Vanilla \\
\hline
\end{tabular}


Table 2. List of taxa recorded in the State Park of Serra do Tabuleiro, Santa Catarina, Brazil. Species are cited for the first time: $1=$ state of the Santa Catarina; $2=$ region studied, data based on lists of the Klein \& al. (1978) and Barros \& al. (2015). Vegetation type (VG): RE=restinga vegetation; $\mathrm{RP}=$ riparian vegetation. Categories of threatened $(\mathrm{CT})$ : $E D=$ endangered, $C E=$ critically endangered, and $\mathrm{VU}=\mathrm{vulnerable}, \mathrm{EX}=\mathrm{presumably}$ extinct. Ecological category (EC): HLC=characteristic holoepiphyte, HLF=facultative holoepiphyte, HEM=hemiepiphyte (Benzing 1990) and TER=terrestrial

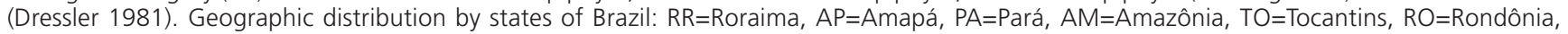
$\mathrm{Pl}=$ Piauí, $\mathrm{CE}=$ Ceará, $\mathrm{RN}=$ Rio Grande do Norte, $\mathrm{PB}=$ Paraíba, $\mathrm{PE}=$ Pernambuco, $\mathrm{AL}=$ Alagoas, $\mathrm{SE}=$ Sergipe, $\mathrm{BA}=\mathrm{B}$ ahia, $\mathrm{DF}=\mathrm{Distrito} F$ Federal, $\mathrm{GO}=$ Goiás, MT=Mato Grosso, MS=Mato Grosso do Sul, ES=Espírito Santo, MG=Minas Gerais, RJ=Rio de Janeiro, SP=São Paulo, PR=Paraná,

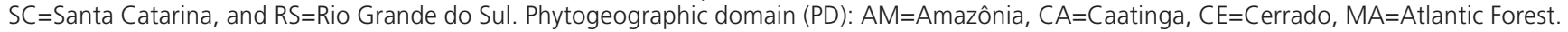
$\mathrm{Di}=$ data deficient

\begin{tabular}{|c|c|c|c|c|c|c|}
\hline Genus and species & Geographic distribution & PD & CT & VG & EC & Voucher \\
\hline $\begin{array}{l}\text { Acianthera ramosa (Barb.Rodr.) } \\
\text { F.Barros }\end{array}$ & $\mathrm{GO}, \mathrm{DF}, \mathrm{MG}, \mathrm{SP}, \mathrm{SC}^{2}$ & CE,MA & & $\mathrm{RE} / \mathrm{RP}$ & $\mathrm{HLC}$ & ABH 57597 -FLOR 39574 \\
\hline $\begin{array}{l}\text { Acianthera saundersiana (Rchb.f.) } \\
\text { Pridgeon \& M.W.Chase }\end{array}$ & $B A, M G, R J, S P, P R, S C, R S$ & $C A, C E, M A$ & & $\mathrm{RP}$ & $\mathrm{HLC}$ & ABH 57598 - FLOR 8661 \\
\hline $\begin{array}{l}\text { Anathallis paranaensis (Schltr.) } \\
\text { Pridgeon \& M.W.Chase }\end{array}$ & $B A, R J, P R, S C, R S$ & CA,MA & & RP & HLC & ABH 57601 \\
\hline $\begin{array}{l}\text { Anathallis sclerophylla (Lindl.) } \\
\text { Pridgeon \& M.W.Chase }\end{array}$ & $R O, C E, P E, B A, R J, S P, P R, S C$ & $C A, C E, M A$ & & $\mathrm{RP}$ & $\mathrm{HLC}$ & ABH 57600 - FLOR 38479 \\
\hline Anathallis sororcula (Schltr.) Luer & $\mathrm{SP}, \mathrm{PR}, \mathrm{SC}^{2}$ & MA & & $\mathrm{RP}$ & $\mathrm{HLC}$ & ABH 57602 \\
\hline Barbosella dusenii (Samp.) Schltr. & $\mathrm{RJ}, \mathrm{SP}, \mathrm{PR}, \mathrm{SC}^{2}$ & MA & & $\mathrm{RP}$ & $\mathrm{HLC}$ & ABH 57603 - FLOR 41825 \\
\hline Bifrenaria aureofulva Lindl. & $\mathrm{BA}, \mathrm{MG}, \mathrm{ES}, \mathrm{RJ}, \mathrm{SP}, \mathrm{PR}, \mathrm{SC}, \mathrm{RS}$ & $C A, C E, M A$ & ED & $\mathrm{RP}$ & $\mathrm{HLC}$ & ABH 57604 \\
\hline Bifrenaria tetragona (Lindl.) Schltr. & $E S, R J, S P, P R, S C, R S$ & MA & ED & $\mathrm{RP}$ & HLF & FLOR 41618 \\
\hline $\begin{array}{l}\text { Brasiliorchis marginata (Lindl.) } \\
\text { R.B.Singer, S.Koehler \& Carnevali }\end{array}$ & $\mathrm{BA}, \mathrm{MG}, \mathrm{ES}, \mathrm{RJ}, \mathrm{SP}, \mathrm{PR}, \mathrm{SC}, \mathrm{RS}$ & MA & & $\mathrm{RP}$ & $\mathrm{HLC}$ & ABH 57608 \\
\hline $\begin{array}{l}\text { Brasiliorchis picta (Hook.) } \\
\text { R.B.Singer, S.Koehler \& Carnevali }\end{array}$ & $M S, M G, E S, R J, S P, P R, S C, R S$ & MA & & $\mathrm{RP}$ & $\mathrm{HLC}$ & FLOR 41819 \\
\hline Brassavola tuberculata Hook. & TO,PA,PE,BA,AL,SE,MG, RJ,SP,PR,SC, RS & $C A, C E, M A$ & & RP & HLC & FLOR 41817 \\
\hline $\begin{array}{l}\text { Bulbophyllum glutinosum } \\
\text { (Barb.Rodr.) Cogn. }\end{array}$ & $M G, E S, R J, S P, P R, S C$ & MA & & $\mathrm{RP}$ & $\mathrm{HLF}$ & ABH 57609 - FLOR 9575 \\
\hline $\begin{array}{l}\text { Campylocentrum aromaticum } \\
\text { Barb.Rodr. }\end{array}$ & $M G, R J, S P, P R, S C^{2}, R S$ & $C E, M A$ & & $\mathrm{RP}$ & $\mathrm{HLC}$ & ABH 57610 \\
\hline Campylocentrum sp. & $\mathrm{Di}$ & $\mathrm{Di}$ & Di & $\mathrm{RP}$ & $\mathrm{HLC}$ & FLOR 9559 \\
\hline $\begin{array}{l}\text { Cyrtopodium flavum (Nees) } \\
\text { Link \& Otto ex Rchb.f. }\end{array}$ & $\mathrm{PB}, \mathrm{PE}, \mathrm{BA}, \mathrm{AL}, \mathrm{SE}, \mathrm{MG}, \mathrm{ES}, \mathrm{RJ}, \mathrm{SP}, \mathrm{PR}, \mathrm{SC}, \mathrm{RS}$ & $C E, M A$ & & RE & TER & ABH 57616 - FLOR 38483 \\
\hline Dichaea cogniauxiana Schltr. & $\mathrm{BA}, \mathrm{MT}, \mathrm{MG}, \mathrm{ES}, \mathrm{RJ}, \mathrm{SP}, \mathrm{PR}, \mathrm{SC}, \mathrm{RS}$ & $C E, M A$ & ED & $\mathrm{RP}$ & $\mathrm{HLC}$ & ABH 57617 \\
\hline Dichaea pendula (Aubl.) Cogn. & $\begin{array}{l}\text { RO,AM,PA, BA, CE, PB, PE, MG, ES, RJ,SP,PR, } \\
\text { SC,RS }\end{array}$ & $\mathrm{AM}, \mathrm{MA}$ & & $\mathrm{RP}$ & $\mathrm{HLC}$ & ABH 57618 - FLOR 38476 \\
\hline Dryadella edwallii (Cogn.) Luer & $M G, S P, R J, P R, S C$ & MA & & RP & $\mathrm{HLC}$ & ABH 57620 - FLOR 9561 \\
\hline Dryadella zebrina (Porsch) Luer & $E S, R J, S P, P R, S C, R S$ & MA & & RP & HLC & ABH 57619 - FLOR 8467 \\
\hline Elleanthus brasiliensis (Lindl.) Rchb.f. & $C E, P E, B A, M G, E S, R J, S P, P R, S C, R S$ & MA & & $\mathrm{RP}$ & HLF & ABH 57621 - FLOR 8647 \\
\hline Encyclia patens Hook. & $\mathrm{PE}, \mathrm{AL}, \mathrm{BA}, \mathrm{SE}, \mathrm{MG}, \mathrm{ES}, \mathrm{RJ}, \mathrm{SP}, \mathrm{PR}, \mathrm{PR}, \mathrm{SC}, \mathrm{RS}$ & $C E, M A$ & & $\mathrm{RP}$ & HLC & ABH 57622 - FLOR 41415 \\
\hline Epidendrum fulgens Brongn. & $R J, S P, P R, S C, R S$ & MA & & RE & HLF & ABH 57623 - FLOR 4138 \\
\hline Epidendrum geniculatum Barb. Rodr. & $E S, R J, S P, P R, S C$ & MA & VU & $\mathrm{RP}$ & HLF & FLOR 22777 \\
\hline Epidendrum latilabre Lindl. & $\mathrm{PA}, \mathrm{AM}, \mathrm{PE}, \mathrm{BA}, \mathrm{MG}, \mathrm{ES}, \mathrm{RJ}, \mathrm{SP}, \mathrm{PR}, \mathrm{SC}, \mathrm{RS}$ & $\mathrm{AM}, \mathrm{MA}$ & & RP & HLC & ABH 57626 \\
\hline Epidendrum paranaense Barb.Rodr. & $\mathrm{BA}, \mathrm{MG}, \mathrm{ES}, \mathrm{RJ}, \mathrm{SP}, \mathrm{PR}, \mathrm{SC}^{2}$ & $\mathrm{CA}, \mathrm{CE}, \mathrm{MA}$ & ED & $\mathrm{RP}$ & $\mathrm{HLC}$ & ABH 57625 - FLOR 8649 \\
\hline Epidendrum proligerum Barb.Rodr. & $A L, P E, B A, M G, E S, R J, S P, P R, S C, R S$ & MA & VU & RP & HLC & ABH 57627 \\
\hline
\end{tabular}


Table 2. (Continued)

\begin{tabular}{|c|c|c|c|c|c|c|}
\hline Genus and species & Geographic distribution & PD & $\mathrm{CT}$ & VG & EC & Voucher \\
\hline Epidendrum secundum Jacq. & $\begin{array}{l}\text { RO,AP,PA,AL,AM,TO,CE,PB,SE,PE,BA,MT, } \\
\text { GO,DF, MG,ES,RJ,SP,PR,SC, RS }\end{array}$ & $A M, C A, C E, M A$ & ED & $\mathrm{RE} / \mathrm{RP}$ & HLF & ABH 57624 - FLOR \\
\hline Eurystyles cotyledon Wawra & $\mathrm{PE}, \mathrm{MG}, \mathrm{ES}, \mathrm{SP}, \mathrm{RJ}, \mathrm{PR}, \mathrm{SC}, \mathrm{RS}$ & MA & & $\mathrm{RP}$ & $\mathrm{HLC}$ & ABH 57628 \\
\hline $\begin{array}{l}\text { Gomesa ciliata (Lindl.) M.W.Chase } \\
\& \text { N.H.Williams }\end{array}$ & $\mathrm{CE}, \mathrm{BA}, \mathrm{MG}, \mathrm{ES}, \mathrm{RJ}, \mathrm{SP}, \mathrm{PR}, \mathrm{SC}, \mathrm{RS}$ & MA & & RP & $\mathrm{HLC}$ & ABH 57629 - FLOR 39562 \\
\hline $\begin{array}{l}\text { Gomesa hookeri (Rolfe) } \\
\text { M.W.Chase \& N.H.Williams }\end{array}$ & $P E, B A, M G, R J, S P, P R, R S, S C^{2}$ & MA & & $\mathrm{RP}$ & $\mathrm{HLC}$ & ABH 57631 - FLOR 39563 \\
\hline $\begin{array}{l}\text { Gomesa praetexta (Rchb.f.) } \\
\text { M.W.Chase \& N.H.Williams }\end{array}$ & $\mathrm{BA}, \mathrm{ES}, \mathrm{RJ}, \mathrm{SP}, \mathrm{PR}, \mathrm{SC}^{2}$ & MA & $\mathrm{VU}$ & RP & $\mathrm{HLC}$ & ABH 57630 \\
\hline $\begin{array}{l}\text { Habenaria pleiophylla Hoehne \& } \\
\text { Schltr. }\end{array}$ & $M G, R J, S P, P R, S C$ & MA & & RE & TER & ABH 57632 - FLOR 9564 \\
\hline $\begin{array}{l}\text { Heterotaxis brasiliensis } \\
\text { (Brieger \& Illg) F.Barros }\end{array}$ & $\mathrm{RN}, \mathrm{PE}, \mathrm{BA}, \mathrm{MG}, \mathrm{ES}, \mathrm{RJ}, \mathrm{SP}, \mathrm{PR}, \mathrm{SC}^{2}, \mathrm{RS}$ & MA & & RE & TER & FLOR 8636 - ABH 57633 \\
\hline Isochilus linearis (Jacq.) R.Br. & $\mathrm{RO}, \mathrm{AL}, \mathrm{CE}, \mathrm{PE}, \mathrm{BA}, \mathrm{DF}, \mathrm{MS}, \mathrm{MG}, \mathrm{ES}, \mathrm{SP}, \mathrm{RJ}, \mathrm{PRSC}, \mathrm{RS}$ & CE,MA & & RP & HLF & FLOR 0215 \\
\hline $\begin{array}{l}\text { Lankesterella ceracifolia } \\
\text { (Barb.Rodr.) Mansf. }\end{array}$ & $\mathrm{BA}, \mathrm{ES}, \mathrm{RJ}, \mathrm{SP}, \mathrm{PR}, \mathrm{SC}, \mathrm{RS}$ & CA,MA & VU & RP & $\mathrm{HLC}$ & ABH 57634 \\
\hline $\begin{array}{l}\text { Lepanthopsis floripecten (Rchb.f.) } \\
\text { Ames }\end{array}$ & $P E, E S, R J, S P, P R, S C$ & MA & EX & RP & $\mathrm{HLC}$ & ABH 57635 \\
\hline Liparis nervosa (Thunb.) Lindl. & $\begin{array}{l}\text { PA,AM,RO, RR,PE,BA,AL,SE,MT,GO,DF, } \\
\text { MSMG,ES,RJ,SP,PR,SC }\end{array}$ & $A M, C A, C E, M A$ & & $\mathrm{RE} / \mathrm{RP}$ & TER & ABH 57636 - FLOR 8642 \\
\hline Malaxis excavata (Lindl.) Kuntze & $\mathrm{PE}, \mathrm{BA}, \mathrm{DF}, \mathrm{MG}, \mathrm{ES}, \mathrm{RJ}, \mathrm{SP}, \mathrm{PR}, \mathrm{SC}, \mathrm{RS}$ & CE,MA & VU & $\mathrm{RP}$ & TER & ABH 57637 - FLOR 8494 \\
\hline Maxillaria bradei Schltr. ex Hoehne & $M G, R J, S P, P R, S C$ & MA & & RP & HLF & ABH 57638 - FLOR 9565 \\
\hline Maxillaria leucaimata Barb.Rodr. & $\mathrm{PA}, \mathrm{AM}, \mathrm{CE}, \mathrm{PE}, \mathrm{BA}, \mathrm{MG}, \mathrm{ES}, \mathrm{RJ}, \mathrm{SP}, \mathrm{PR}, \mathrm{SC}$ & AM,MA & & RP & $\mathrm{HLC}$ & ABH 57639 \\
\hline Maxillaria lindleyana Schltr. & $M G, E S, R J, S P, P R, S C$ & MA & & RP & HLF & FLOR 9566 \\
\hline Maxillaria ochroleuca Lodd. ex Lindl. & $\mathrm{RR}, \mathrm{PE}, \mathrm{AL}, \mathrm{BA}, \mathrm{MG}, \mathrm{ES}, \mathrm{RJ}, \mathrm{SP}, \mathrm{PR}, \mathrm{SC}$ & $A M, C E, M A$ & & $\mathrm{RP}$ & $\mathrm{HLF}$ & ABH 57640 - FLOR 9576 \\
\hline Mesadenella cuspidata (Lindl.) Garay & $\mathrm{MT}, \mathrm{CE}, \mathrm{PE}, \mathrm{GO}, \mathrm{DF}, \mathrm{MGES}, \mathrm{RJ}, \mathrm{SP}, \mathrm{PR}, \mathrm{SC}, \mathrm{RS}$ & CE,MA & & $\mathrm{RE} / \mathrm{RP}$ & TER & ABH 57642 - FLOR 9577 \\
\hline Myoxanthus exasperatus (Lindl.) Luer & $\mathrm{PE}, \mathrm{BA}, \mathrm{MG}, \mathrm{ES}, \mathrm{RJ}, \mathrm{SP}, \mathrm{PR}, \mathrm{SC}$ & CE,MA & & RP & HLF & ABH 67643 - FLOR 43893 \\
\hline Octomeria alexandri Schltr. & $\mathrm{PE}, \mathrm{BA}, \mathrm{ES} . \mathrm{RJ}, \mathrm{SP}, \mathrm{SC}^{1,2}, \mathrm{RS}$ & CE,MA & EX & $\mathrm{RP}$ & HLF & ABH 57644 - FLOR 9678 \\
\hline $\begin{array}{l}\text { Octomeria chloidophylla (Rchb.f.) } \\
\text { Garay }\end{array}$ & $\mathrm{RJ}, \mathrm{SC}$ & MA & & RP & $\mathrm{HLF}$ & ABH 57649 \\
\hline Octomeria crassifolia Lindl. & $\mathrm{BA}, \mathrm{MT}, \mathrm{MG}, \mathrm{ES}, \mathrm{RJ}, \mathrm{SP}, \mathrm{PR}, \mathrm{SC}, \mathrm{RS}$ & MA & & $\mathrm{RP}$ & $\mathrm{HLC}$ & ABH 67652 - FLOR 9568 \\
\hline Octomeria diaphana Lindl. & $\mathrm{MG}, \mathrm{ES}, \mathrm{RJ}, \mathrm{SP}, \mathrm{PR}, \mathrm{SC}, \mathrm{RS}$ & MA & & $\mathrm{RP}$ & $\mathrm{HLC}$ & ABH 57653 \\
\hline Octomeria gracilis Lodd. ex Lindl. & $M G, E S, R J, S P, P R, S C, R S$ & MA & & RP & $\mathrm{HLC}$ & ABH 57648 - FLOR 9567 \\
\hline Octomeria grandiflora Lindl. & $\begin{array}{l}\text { RR,AP,PA,AM,AC, MA, PB, BA, MG, ES, RJ,SP, } \\
P R, S C\end{array}$ & $A M, C A, C E, M A$ & & RP & $\mathrm{HLC}$ & ABH 57646 - FLOR 8628 \\
\hline Octomeria juncifolia Barb.Rodr. & $M G, E S, R J, S P, P R, S C, R S$ & MA & & RP & $\mathrm{HLC}$ & ABH 57651-FLOR 8632 \\
\hline Octomeria oxychela Barb.Rodr. & $\mathrm{MS}, \mathrm{MG}, \mathrm{ES}, \mathrm{RJ}, \mathrm{SP}, \mathrm{PR}, \mathrm{SC}^{2}, \mathrm{RS}$ & $C E, M A$ & & RP & $\mathrm{HLC}$ & ABH 57650 \\
\hline Octomeria riograndensis Schltr. & $\mathrm{PR}, \mathrm{SC}, \mathrm{RS}$ & MA & & RP & $\mathrm{HLC}$ & ABH 57645 \\
\hline Octomeria umbonulata Schltr. & $\mathrm{SP}, \mathrm{PR}, \mathrm{SC}, \mathrm{RS}$ & MA & & RP & $\mathrm{HLC}$ & ABH 57647 \\
\hline $\begin{array}{l}\text { Oeceoclades maculata } \\
\text { (Lindl.) Lindl. }\end{array}$ & $\begin{array}{l}\text { RR,PA,AM,TO,AC,RO,MA,PI,CE,RN, } \\
\text { PB,PE,AL,SE,MT, GO,DF,MS,MG, } \\
\mathrm{BA}, \mathrm{ES}, \mathrm{RJ}, \mathrm{SP}, \mathrm{PR}, \mathrm{SC}^{2}, \mathrm{RS}\end{array}$ & $A M, C A, C E, M A$ & & $\mathrm{RE} / \mathrm{RP}$ & TER & ABH 57654 - FLOR 8666 \\
\hline $\begin{array}{l}\text { Ornithidium pendens (Pabst) } \\
\text { Senghas }\end{array}$ & $\mathrm{AM}, \mathrm{RO}, \mathrm{MG}, \mathrm{ES}, \mathrm{RJ}, \mathrm{SP}, \mathrm{PR}, \mathrm{SC}^{, 2}{ }^{2}, \mathrm{RS}$ & AM,MA & & $\mathrm{RP}$ & $\mathrm{HLC}$ & ABH 57655 \\
\hline Ornithocephalus myrticola Lindl. & $\mathrm{BA}, \mathrm{MG}, \mathrm{ES}, \mathrm{RJ}, \mathrm{SP}, \mathrm{PR}, \mathrm{SC}, \mathrm{RS}$ & CE,MA & CE & RP & $\mathrm{HLC}$ & ABH 57656 - FLOR 41649 \\
\hline $\begin{array}{l}\text { Pabstiella campestris (Barb.Rodr.) } \\
\text { Luer }\end{array}$ & $\mathrm{MG}, \mathrm{RJ}, \mathrm{SP}, \mathrm{PR}, \mathrm{SC}, \mathrm{RS}$ & MA & & $\mathrm{RP}$ & $\mathrm{HLC}$ & FLOR 8613 \\
\hline $\begin{array}{l}\text { Pabstiella fusca (Lindl.) Chiron \& } \\
\text { Xim.Bols. }\end{array}$ & $\mathrm{BA}, \mathrm{MG}, \mathrm{ES}, \mathrm{RJ}, \mathrm{SP}, \mathrm{PR}, \mathrm{SC}, \mathrm{RS}$ & CE,MA & & RP & $\mathrm{HLC}$ & ABH 57657 \\
\hline Pabstiella matinhensis (Hoehne) Luer & $\mathrm{PR}, \mathrm{SC}^{2}, \mathrm{RS}$ & MA & & RP & $\mathrm{HLC}$ & ABH 57664 \\
\hline $\begin{array}{l}\text { Paradisanthus micranthus } \\
\text { (Barb.Rodr.) Schltr. }\end{array}$ & $\mathrm{BA}, \mathrm{ES}, \mathrm{RJ}, \mathrm{SP}, \mathrm{PR}, \mathrm{SC}$ & MA & & RP & TER & ABH 57658 \\
\hline Pelexia sp. & Di & Di & & RE & TER & ABH 57659 \\
\hline $\begin{array}{l}\text { Phloeophila nummularia (Rchb.f.) } \\
\text { Garay }\end{array}$ & $M G, R J, S P, S C$ & MA & & RP & $\mathrm{HLC}$ & ABH 57660 \\
\hline
\end{tabular}


Table 2. (Continued)

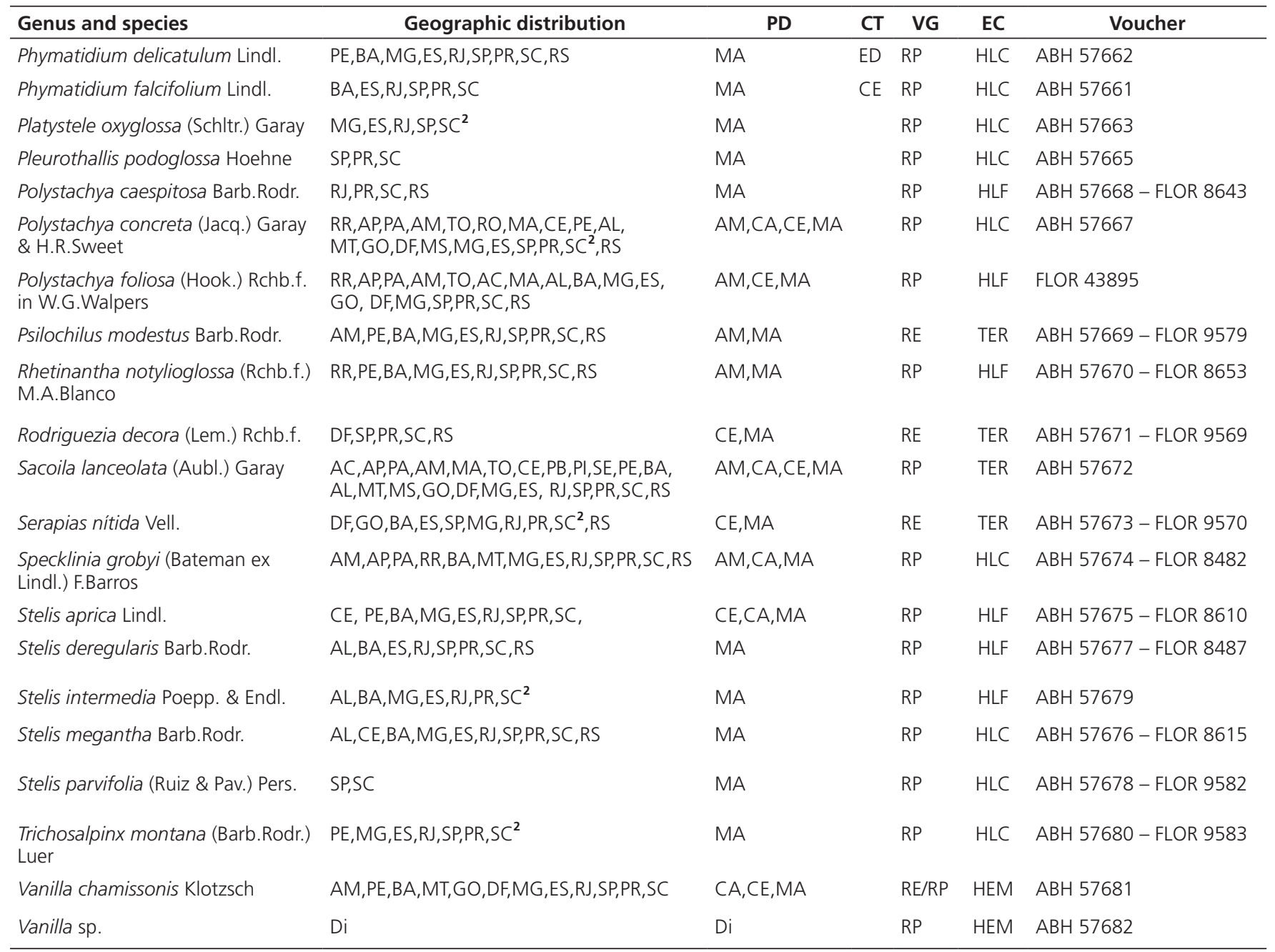

A. ramosa, E. secundum, L. nervosa, $M$. cuspitata, O. maculata and $V$. chamissonis, occur in both habitats (Table 2).

The predominant ecological category was the characteristic holoepiphyte (HLC) (Table 2), represented by 55 species $(60 \%)$, which was followed by facultative holoepiphyte (HLF) with 21 species $(23 \%$ ) and terrestrial (TER) with 14 species $(15 \%)$. The hemiepiphytes were less common, with only two species (2\%) (Fig. 2), both belonging to the genus Vanilla (Table 2).

Characteristic holoepiphytes (HLC) and facultative holoepiphytes (HLF) were better represented in riparian vegetation $(n=53 ; 58 \%$ and $n=18 ; 20 \%$, respectively. Terrestrial species (TER) were better represented in resting a vegetation $(\mathrm{n}=10 ; 11 \%)$. Characteristic holoepiphyte (HLC) were not exclusive in restinga vegetation (Table 2 \& Fig. 2).

\section{DISCUSSION}

The richness of orchid species found in the present study can be considered high when compared to other studies on Orchidaceae in different regions of Brazil. In the Southern region, Rocha \& Waechter (2006) studied terrestrial orchids in the area of resting a vegetation and found 42 species distributed in 24 genera. In the same area, Buzatto \& al. (2007) cited 50 species and 35 genera from an area of riparian vegetation. In the Southeast region, Fraga \& Peixoto (2004) cited 73 species distributed in 41 genera, in the restinga vegetation; and Cunha \& Forzza (2007) in an area covered by Atlantic Forest and restinga vegetations found 26 species in 18 genera. In other regions, Pansarin \& Pansarin (2008) recorded 125 species in an area mesophytic-semideciduous forest, and Menini-Neto \& al. (2009) cited 89 species in three areas of Atlantic Forest, montane forest and semi-deciduous forest at different altitudes.

For the state of Santa Catarina, no recent floristic surveys on Orchidaceae are available and those extant are outdated, a fact that makes further precise comparisons extremely difficult. Nonetheless, if the results are compared to that of Klein \& al. (1978), it is noticeable that the species richness found here appears to be not very much representative. However, some relevant factors must be considered for a better understanding of the results. The studied area was limited to a few remnants of herbaceous and shrubby restinga vegetation still found in the region, and the sampling in riparian vegetation was restricted by up to five meters in height on the phorophytes. In fact, our study though performed in the same region covered a smaller area when compared to that of Klein \& al. (1978), which was much wider in 
Ecological Category

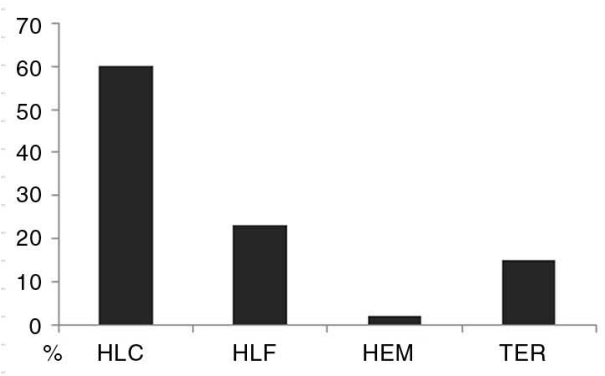

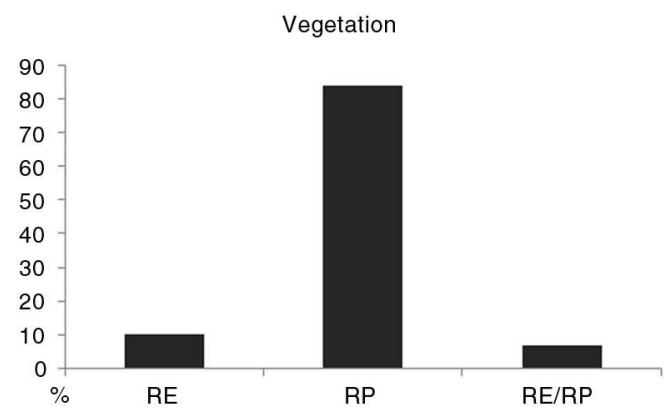

Ecological Category $x$ Vegetation

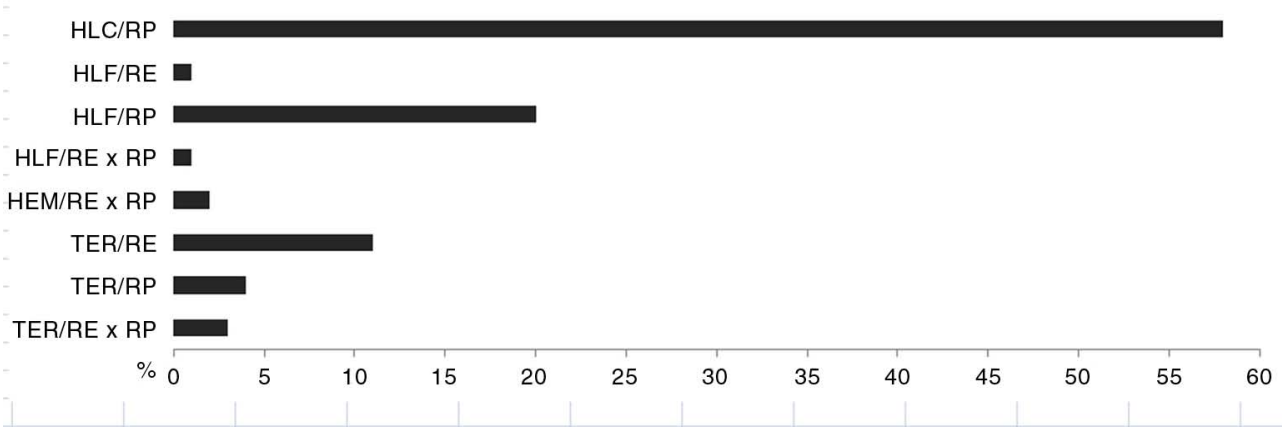

Fig. 2. Orchids recorded in restinga (RE) and riparian vegetation (RP) in the Atlantic Forest, Santa Catarina, Brazil. Data expressed in percentages by their ecological category and comparison of the vegetation between ecology category, based on the total number of species recorded ( $\mathrm{n}=92$ ): $\mathrm{HLC}=\mathrm{characteristic}$ holoepiphyte, HLF=facultative holoepiphyte, HEM=hemiepiphyte (Benzing 1990) and TER=terrestrial (Dressler 1981). Characteristic holoepiphyte (HLC) were not exclusive in restinga vegetation.

both geographic and ecologic terms. However, we report 20 new records for the same region of the study, demonstrating that there is still a great diversity. Undoubtedly, if the studied area were greater, including other types of vegetation, and also the canopy of the forest, the number of species recorded probably would be much greater.

The State Park of Serra do Tabuleiro was created to preserve an area that had already undergone processes of environmental deface, generated by logging, agriculture and urbanizing activities. Certainly, when taken into account the study of Orchidaceae by Fraga \& Peixoto (2004) and Rocha $\&$ Waechter (2006), made in areas of restinga vegetation in the state of Rio Grande do Sul, also in southern Brazil, the specific diversity found in our study is extremely low. The fragmentation and haphazard occupation of coastal areas are identified by those authors as negative factors for the conservation of restinga vegetation and for orchids themselves. According to Rocha \& Waechter (2006), when performed in a short period of time studies on herbaceous terrestrial species in the restinga vegetation probably underestimate the total specific richness of Orchidaceae. This mainly occurs because of the difficulty to observe many species that often are represented by tiny and isolated individuals, with low frequency and/or without leaves during anthesis.

Orchids occur in up to two phytogeographic domains, although the great majority is found only in Atlantic Forest. It is believed that for species of Atlantic Forest there would be a continuum in their distributions in the N-S direction (Klein, 1980). However, this is not always the rule. Some species such as $P$. falcifolium, $P$. nummularia and $S$. parvifolia occur without gaps up to the state of São Paulo, and they only reappear in the state of Santa Catarina to the south. It is also possible that the lack of detailed studies on Orchidaceae in the states of Paraná and Santa Catarina make difficult a more accurate phytogeographic understanding of their distribution patterns. Furthermore, our results show that $37 \%$ of the studied species are found continuously from the northern states southwards to Santa Catarina, but they do not reach the state of Rio Grande do Sul (in the very South of Brazil). According to Klein (1978; 1980), Serra do Tabuleiro is the most important phytogeographic barrier in southern Brazil impeding that many plants species can move in a north-south direction. In fact, Orchidaceae are among the leading families that are not able to cross this geographic boundary, so that one-third of the orchid species growing in the north, disappear in the southern part of that mountain range (Klein, 1980).

Although up to seventeen of the studied species are listed on any of the Brazilian regional red lists of endangered species list, only one of them is included in the most recent list of endangered species of the Brazilian flora, published by Ministério do Meio Ambiente (MMA, 2015). Nonetheless, at a local scale a number of those species (as well as others not included in the lists) can be in serious concern. It is therefore urgent to generate regional and local lists as an effective conservation tool for threatened species. Provided that many changes have occurred in the last 18 years that affected areas of natural vegetation through the whole country, it can be assumed that the threat degree for many species should also change. Galindo-Leal \& Câmara (2005) suggested that the entire Biome of Atlantic Forest is seriously threatened. According to a survey of Fundação SOS Mata Atlântica 
(2013), the deforestation rate in the state of Santa Catarina increased up to $7 \%$ between 2000 and 2005. Although a reduction in that rate was recorded between 2008 and 2010, deforestation continues to be a significant threat in the state. In relation to the forest remnants of 2008 , currently $0.17 \%$ (3626 ha) of forests have already been lost, the restinga vegetation being the most seriously affected with $0.10 \%$ ( 75 ha) of its cover deforested (Fundação SOS Mata Atlântica, 2013).

The characteristic holoepiphyte was the most common ecologic category among the studied specimens and this agrees with other studies on Orchidaceae in Brazil (Barros, 2004; Fraga \& Peixoto, 2004; Cunha \& Forzza, 2007; Buzzato \& al., 2007; Menini-Neto \& al., 2009, Medeiros \& Jardim, 2011). According to Benzing (1990), Orchidaceae are well known for their success in tree colonization, being orchids about two out of three epiphytes on every tree. However, many of them behave as facultative holoephiphytes, commonly also found growing on rocks on the banks of rivers and in small river islands, as demonstrated by the present study. Similarly, Falkenberg (2003) describes that the epiphytic habitats can easily be compared to the rocky habitats since both share a similar ecological situation, and in many cases epiphytic species can even grow best on rocks, if light conditions are satisfactory.

In terms of conservation, it is of paramount importance to preserve all diversity of vegetation types found in the State Park of Serra do Tabuleiro this would ensure the maintenance of different habitats suitable for colonization and perpetuation of orchid populations. Conservation figures such as 'plant micro-reserves' (cf. Laguna, 2001; Laguna $\&$ al., 2004) would be useful for that purpose, since they are small plots that require active management to maintain vegetation spots unchangeable along time, therefore avoiding community (and also species) successional replacement. This is especially relevant in the riparian vegetation, in which most species are found, including several threatened species. Indeed, the orchid community from the restinga vegetation shows lower diversity rates when compared to riparian vegetation. However, the history of environmental degradation endured by the studied coastal area of the State Park of Serra do Tabuleiro may be influencing negatively the current floristic composition of Orchidaceae in the restinga vegetation.

\section{ACKNOWLEDGEMENTS}

We thank José Carlos Cristóbal (ABH) and Silvia Venturi (FLOR) for their help in preparing and recording specimens for their herbaria. The first author was supported by CAPES (Coordenação de Aperfeiçoamento de Pessoal de Nível Superior) - Postdoctoral scholarship (Proc. 5328-09-8).

\section{REFERENCES}

Barros, F. 2004. Distribuição geográfica de orquídeas do Planalto Central do Brasil. In: Barros, F. \& Kerbauy, G.B. (eds.), Orquidologia sul-americana: uma compilação científica: 147-153. São Paulo.

Barros, F., Vinhos, F., Rodrigues, V.T., Barberena, F.F.V.A., Fraga, C.N., Pessoa E.M., Forster, W., Menini Neto, L., Furtado, S.G., Nardy, C., Azevedo, C.O., Guimarães, L.R.S. 2015 [continuously updated]. Orchidaceae. Lista de Espécies da Flora do Brasil on line. Jardim Botânico do Rio de Janeiro. http://floradobrasil.jbri.gov.br/jabot/floradobrasil/FB179

Battilani, J.L., Scremin-Dias, E. \& Souza, A.L.T. 2005. Fitossociologia de um trecho da mata ciliar do rio da Prata, Jardim, M.S., Brasil. Acta Botanica Brasilica 19: 597-608. http://dx.doi.org/10.1590/S0102 33062005000300021
Benzing, D.H. 1990. Vascular epiphytes. General biology and related biota. Cambridge University Press, New York. http://dx.doi.org/10.1017/ CBO9780511525438

Buzatto, C.R., Freitas, E.M., Silva, A.P.M. \& Lima, L.F.P. 2007. Levantamento florístico das Orchidaceae ocorrentes na Fazenda São Maximiano, Município de Guaíba, Rio Grande do Sul. Revista Brasileira de Biociências 5: $19-25$.

Chase, M.W., Cameron, K.M., Barrett, R.L. \& Freudenstein, J.V. 2003. DNA data and Orchidaceae systematics: a new phylogenetic classification. In: Dixon, K.W., Kell, S.P., Barrett, R.L. \& Cribb, P.J. (eds.), Orchid Conservation Natural History Publications: 69-89. Sabah.

Cunha, M.F.B. \& Forzza, R.C. 2007. Orchidaceae no Parque Natural Municipal da Prainha, RJ, Brasil. Acta Botanica Brasilica 21: 383-400. http://dx.doi.org/10.1590/S0102-33062007000200013

Dressler, R.L. 1981. The orchids. Natural history and classification. Harvard University Press, Cambridge.

Dressler, R.L. 1993. Phylogeny and classification of the orchid family. Dioscorides, Portland.

Falkenberg, D.B. 1999. Aspectos da flora e da vegetação secundária da restinga de Santa Catarina, Sul do Brasil. Insula 28: 1-30.

Falkenberg, D.B. 2003. Matinhas nebulares e vegetação rupicola dos Aparados da Serra Geral (SC/RS), Sul do Brasil. Doctoral thesis, Universidade Estadual de Campinas, São Paulo.

Fraga, C.N. \& Peixoto, A.L. 2004. Florística e ecologia das Orchidaceae das restingas do Estado do Espírito Santo. Rodriguésia 55: 5-20.

Fundação SOS Mata Atlântica. (ed.). 2013. Atlas dos remanescentes Florestais da Mata Atlântica (2008-2010). http://mapas.sosma.org.br/ site_media/download/atlas_2008-10_relatorio_final.pdf

Galindo-Leal, C. \& Câmara, I.G. 2005. Status do Hotspot Mata Atlântica: uma síntese. In: Galindo-Leal, C. \& Câmara, I.G. (eds.). Mata Atlântica: biodiversidade, ameaças e perspectivas: 3-12. Belo Horizonte.

IBGE. 2014. Instituto Brasileiro de Geografia e Estatística. Base Cartográfica Contínua do Brasil 1: 1000000. http://downloads.ibge.gov.br/downloads_ geociencias.htm

INMET. (ed.). 2011. Instituto Nacional de Meteorologia. Banco de dados meteorológicos. http://www.inmet.gov.br/projetos/rede/pesquisa/

IPEMA. (ed.). 2007. Instituto de Pesquisas da Mata Atlântica/Instituto de Espécies Ameaçadas. Lista de espécies ameaçadas do Espírito Santo (2007). http://www.biodiversitas.org.br/listas-mg/ES-especies-ameacadas.pdf

IPNI. 2015. The International Plant Names Index. Published on the Internet. http://www.ipni.org [continuously updated]

Kageyama, P. \& Gandara, F.B. 2000. Recuperação de áreas ciliares. In Rodrigues, R.R. \& Leitão Filho, H.F. (eds.). Matas ciliares: conservação e recuperação: 249-269. São Paulo.

Klein, R.M. 1978. Mapa fitogeográfico do Estado de Santa Catarina. Flora Ilustrada Catarinense, Itajaí.

Klein, R.M. 1980. Ecologia da flora e vegetação do vale do Itajaí. Sellowia 31/32: 9-389.

Klein, R.M. 1981. Fisionomia, importância e recursos da vegetação do Parque Estadual da Serra do Tabuleiro. Sellowia 33: 5-54.

Klein, R.M. 1990. Espécies raras ou ameaçadas de extinção do estado de Santa Catarina. Instituto Brasileiro de Geografia e Estatística, Rio de Janeiro.

Klein, R.M., Bresolin, A. \& Reis, A. 1978. Distribuição de orquídeas da Ilha de Santa Catarina e arredores. Insula 9: 3-29.

Laguna, E. 2001. The micro-reserves as a tool for conservation of threatened plants in Europe. [Nature and Environment 121]. Council of Europe Publishing, Strasbourg.

Laguna, E., Deltoro, V., Pérez-Botella, J., Pérez-Rovira, P., Serra, L., Olivares, A. \& Fabregat, C. 2004. The role of small reserves in plant conservation in a region of high diversity in eastern Spain. Biological Conservation 119: 421-426. http://dx.doi.org/10.1016/j.biocon.2004.01.001

Medeiros, T.D.S. \& Jardim, M.A.G. 2011. Distribuição vertical de orquídeas epífitas na Área de Proteção Ambiental (APA) Ilha do Combu, Belém, Pará, Brasil. Revista Brasileira de Biociências 9: 33-38.

Menini-Neto, L., Forzza, R.C. \& Zappi, D. 2009. Angiosperm epiphytes as conservation indicators in forest fragments: A case study from southeastern Minas Gerais, Brazil. Biodiversity and Conservation 18 3785-3807. http://dx.doi.org/10.1007/s10531-009-9679-2

Miller, D. \& Warren, R. 1996. Orquídeas do Alto da Serra da Mata Atlântica Pluvial do Sudeste do Brasil. Salamandra, Rio de Janeiro.

Miller, D., Warren, R., Miller, I.M. \& Seehawer, H. 2006. Serra dos Órgãos sua bistória e suas orquídeas. Scart, Nova Friburgo.

MMA. 2015. Ministério do Meio Ambiente. Mapa dos biomas brasileiros. http://mapas.mma.gov.br/mapas/aplic/probio/datadownload.htm 
Pabst, G.F.. \& Dungs, F. 1975. Orchidaceae Brasilienses. Brücke-Verlag Kurt Schmersow, Hildesheim.

Pabst, G.F.J. \& Dungs, F. 1977. Orchidaceae Brasilienses. Brücke-Verlag Kurt Schmersow, Hildesheim.

Pansarin, E.R. \& Pansarin, L.M. 2008. A família Orchidaceae na Serra do Japi, São Paulo, Brasil. Rodriguésia 59: 99-111.

Pereira, G., Ferreira, N.J., Moraes, E.C., Cardozo, F.S. \& Freitas, S.R. 2009 Análise das áreas queimadas e das emissões dos gases do efeito estufa no Parque Estadual da Serra do Tabuleiro, Santa Catarina. Geosul 24: 113-130. http://dx.doi.org/10.5007/2177-5230.2009v24n47p113

Rocha, F.S. \& Waechter, J.L. 2006. Sinopse das Orchidaceae terrestres ocorrentes no litoral norte do Rio Grande do Sul, Brasil. Acta Botanica Brasilica 20: 71-86. http://dx.doi.org/10.1590/S0102-33062006000100008

Rocha, F.S. \& Waechter, J.L. 2010. Ecological distribution of terrestrial orchids in a South Brazilian Atlantic region. Nordic Journal of Botany 28: 112-118. http://dx.doi.org/10.1111/j.1756-1051.2009.00500.x

Rodrigues, R.R. \& Gandolfi, S. 2001. Conceitos, tendências e ações para a recuperação de florestas ciliares. In: Rodrigues, R.R. \& Leitão Filho, H.F. (eds.), Matas Ciliares, Conservação e Recuperação: 235-247. São Paulo.

Scherer, A., Maraschin-Silva, F. \& Baptista, L.R.M. 2005. Florística e estrutura do componente arbóreo de matas de restinga arenosa no Parque Estadual de Itapuã, RS, Brasil. Acta Botanica Brasilica 19: 717-726. http://dx.doi.org/10.1590/S0102-33062005000400006
SEMA. 1995. Secretaria Municipal do Meio Ambiente. Lista vermelha de plantas ameaçadas de extinção no estado do Paraná. Secretaria Municipal do Meio Ambiente/Cooperação Técnica Alemã, Curitiba.

SEMA. (ed.). 2004. Secretaria Municipal do Meio Ambiente. Lista of cial das espécies da flora do estado de São Paulo ameaçadas de extinção (2004). http://www.ibot.sp.gov.br/resolucao_sma48/resolucao48.htm [accessed: January-2013].

SEMA. (ed.). 2014. Secretaria Municipal do Meio Ambiente. Lista oficial das espécies de plantas ameaçadas de extinção no estado do Rio Grande do Sul (2002). http://www.sema.rs.gov.br/sema/html/pdf/especies-ameacadas [accessed: January-2013].

Stancik, J.F. 2004. O gênero Epidendrum L. (Orchidaceae) no Estado do Paraná, Brasil. Ciências Biológicas. Universidade Federal do Paraná, Curitiba.

Thiers, B. 2015. Index Herbariorum: A global directory of public herbaria and associated staff. New York Botanical Garden's Virtual Herbarium. http:// sweetgum.nybg.org/ih/ [continuously updated].

USGS, 2015. U.S. [continuously updated]. U.S. Geological Survey. Landsat-8: http://earthexplorer.usgs.gov/

Associate Editor: Estefan Gleissberg Received: 03-III-2013 Accepted: 2-X-2014 
APPENDIX 1. Characteristics of the collection sites

\begin{tabular}{|c|c|c|c|c|c|c|}
\hline \multicolumn{2}{|c|}{ Sites } & \multicolumn{2}{|c|}{ Coordinates } & \multirow{2}{*}{$\begin{array}{c}\text { Elevation } \\
0-10 \mathrm{~m}\end{array}$} & \multirow{2}{*}{$\frac{\text { Sampling date }}{23-|1|-2010}$} & \multirow{2}{*}{$\begin{array}{l}\text { Voucher } \\
\text { ABH-57597 - ABH-57616 - ABH-7629 - ABH- } \\
\text { 57632 - ABH-57654 - ABH-57673 - ABH-57681 - } \\
\text { FLOR-38483 - FLOR-39564 - FLOR-38642 - FLOR- } \\
\text { 39570 - FLOR-39577 - FLOR-4138 }\end{array}$} \\
\hline 1 & $\begin{array}{l}\text { BRAZIL. Palhoça: } \\
\text { Mouth river Maciambú, } \\
\text { Sonho beach. Restinga } \\
\text { vegetation. }\end{array}$ & $27^{\circ} 49^{\prime} 30.3^{\prime \prime} \mathrm{S}$ & $48^{\circ} 35^{\prime} 34.5^{\prime \prime} \mathrm{W}$ & & & \\
\hline 2 & $\begin{array}{l}\text { BRAZIL. Palhoça: Mouth } \\
\text { the river Maciambú, } \\
\text { Sonho beach. Restinga } \\
\text { vegetation. }\end{array}$ & $27^{\circ} 49^{\prime} 27.5^{\prime \prime} \mathrm{S}$ & $48^{\circ} 35^{\prime} 35.6^{\prime \prime} \mathrm{W}$ & 0-10 m & $10-I V-2010$ & $\begin{array}{l}\text { ABH-57611 - ABH-57623 - ABH-57631 - ABH- } \\
\text { 57633- ABH-57659 - ABH-57671 - FLOR-8636 - } \\
\text { FLOR-39574 - FLOR-39562 - FLOR-39569 }\end{array}$ \\
\hline 4 & $\begin{array}{l}\text { BRAZIL. Palhoça: River } \\
\text { Cachoeira do Amarinho. } \\
\text { Riparian vegetation. }\end{array}$ & $27^{\circ} 43^{\prime} 33.3^{\prime \prime} \mathrm{S}$ & $48^{\circ} 41^{\prime} 46.0^{\prime \prime} \mathrm{W}$ & $75-100 \mathrm{~m}$ & $1-V-2010$ & $\begin{array}{l}\text { ABH-57601 - ABH-57609 - FLOR-39575 - ABH- } \\
39559 \text { - FLOR-44005 - ABH-57618 - ABH-38649 - } \\
\text { ABH-57624 - ABH } 57636 \text { - FLOR-39565 - ABH- } \\
\text { 57640 - ABH-57642 - ABH-57652 - ABH-57679 - } \\
\text { ABH-5762 - FLOR-39566 - FLOR-43893 - FLOR- } \\
\text { 39568 - FLOR-38628 - FLOR-43895 - FLOR-38653 - } \\
\text { FLOR-38610 - FLOR-38615 - ABH-57676 - FLOR- } \\
\text { 41636 - FLOR-41649 }\end{array}$ \\
\hline 5 & $\begin{array}{l}\text { BRAZIL. Palhoça - } \\
\text { Santo Amaro da } \\
\text { Imperatriz: River } \\
\text { Cachoeira do Amarinho. } \\
\text { Riparian vegetation }\end{array}$ & $27^{\circ} 44^{\prime} 05.0^{\prime \prime} \mathrm{S}$ & $48^{\circ} 41^{\prime} 46.0^{\prime \prime} \mathrm{W}$ & $200-250 \mathrm{~m}$ & $3-V-2010$ & $\begin{array}{l}\text { ABH-57604 - FLOR-38475 - ABH-57606 - FLOR- } \\
38476 \text { - FLOR-39561 - FLOR-57625 - ABH-57638 - } \\
\text { ABH-57639 - ABH-57646 - FLOR-38666- } \\
\text { ABH-57665 - ABH-57667 - FLOR-57667 - FLOR- } \\
\text { 39579 - ABH-57669 - ABH-57670 - FLOR-41817 - } \\
\text { FLOR-44005 - FLOR-43895 }\end{array}$ \\
\hline 6 & $\begin{array}{l}\text { BRAZIL. Santo Amaro } \\
\text { da Imperatriz: Tributary } \\
\text { river Vargem do Braço. } \\
\text { Riparian vegetation. }\end{array}$ & $27^{\circ} 47^{\prime} 45.5^{\prime \prime} \mathrm{S}$ & $48^{\circ} 50^{\prime} 01.4^{\prime \prime} \mathrm{W}$ & $345-385 m$ & $17-V-2010$ & $\begin{array}{l}\text { ABH-57602 - FLOR-3922 - FLOR-39559 - ABH- } \\
57613 \text { - ABH-57617 - ABH-57620 - FLOR-38467 - } \\
\text { ABH-57619 - ABH-57626 - FLOR-9563 - ABH-57649 - } \\
\text { ABH-57648 - ABH-57650 - ABH-57657 - ABH- } \\
57664 \text { - ABH-57661 - ABH-57668 - FLOR-38643 - } \\
\text { ABH-57672 - FLOR-38487 - ABH-57677 - ABH- } \\
57680 \text { - FLOR-41819 - FLOR-41415 - FLOR-41825 }\end{array}$ \\
\hline
\end{tabular}

